years, with secondary prevention patients automatically considered high risk. The guidelines in Scotland, and elsewhere, state that statins should be offered in secondary prevention regardless of their plasma lipid concentration and that annual monitoring of lipid concentration represents good practice. This descriptive analysis investigated the demographics of a secondary prevention population, their estimated adherence to statin medication and whether targets for cholesterol reduction were achieved.

Methods Data was extracted from the NHS Greater Glasgow and Clyde (NHS GGC) Safe Haven for all patients with a record of a non-fatal myocardial infarction (MI) occurring between 1st March 2008 and 1st March 2014, together with their demographics, lipid concentrations, and statin dispensing records up to 1st March 2017. Estimated adherence for each year post MI was calculated from the dispensing records using the Medication Possession Ratio (MPR) and averaged across the years available for each patient. Descriptive statistics were presented using raw counts and percentages, and means and standard deviations, for categorical and continuous variables respectively. Logistic regression examined associations between adherence and cholesterol targets, and adherence and mortality, and were also adjusted for age, sex, year of MI and deprivation quintile, with results presented as odds ratios (OR).

Results In the population, 11,568 patients had a previous non-fatal MI, and were predominantly male (7,002, 60.5\%), had a mean age at admission of 66.9 years (SD: 13.9), and approximately a third died before 1st March 2017 (4,053, $35.0 \%$ ). 10,469 patients had at least one year's follow-up allowing average adherence to be estimated and one third $(3,360,32.1 \%)$ had an average statin adherence $<80 \%$. Patients with $<80 \%$ adherence were associated with lower odds of achieving target $40 \%$ non-HDL reduction (OR: 0.387 [95\% CI: 0.336, 0.446]), which were unaltered after adjustment (OR 0.392 [95\% CI: 0.339, 0.452]). These patients were also associated with higher odds of mortality (OR: 1.717 [95\% CI: 1.571, 1.877]), with some attenuation following adjustment (OR: 1.653 [95\% CI: 1.486, 1.839]).

Conclusion In the NHS GGC post MI population, patients with lower statin adherence were associated with lower odds of achieving cholesterol targets set by current guidelines and higher odds of mortality. However, a large proportion of patients achieved targets and had excellent adherence, questioning the need for regular lipid monitoring.

\section{OP17 BIOPSYCHOSOCIAL DETERMINANTS OF CARDIOVASCULAR AND DEMENTIA RISK: AN EXAMINATION OF DIFFERENTIALS IN A REPRESENTATIVE STUDY OF THE ENGLISH POPULATION}

D Cadar*, A Steptoe. Behavioural Science and Health, University College London, London, UK

\subsection{6/jech-2019-SSMabstracts. 17}

Background Growing evidence supports a strong and likely causal association between cardiovascular disease (CVD) and dementia incidence. Several cardiometabolic risk factors including smoking, sedentary behaviour, hypertension, and diabetes are common to both conditions, but less clear is the predictive value of inflammatory markers, alcohol consumption or depression. We investigated the congruity and difference across $10 \mathrm{CVD}$ and dementia risk factors in a representative cohort of the English population.
Methods Data come from the English Longitudinal Study of Ageing, an ongoing, open, prospective cohort study. CVD was defined as the fatal and non-fatal myocardial infarction, angina pectoris and stroke. Dementia was determined using doctordiagnosis combined with a score above the threshold of 3.38 on the Informant Questionnaire on Cognitive Decline in the Elderly. A triangulation method was also used to derive these outcomes using the Hospital Episode Statistics (HES) records. We investigated 10 risk factors: alcohol, smoking, sedentary behaviour, hypertension, diabetes, depressive symptoms, obesity (defined as $\geq 30$ body mass index (BMI)), HDL/total cholesterol and inflammatory markers (serum fibrinogen and C-reactive protein [CRP]) in 3,981 men and women, free of CVD or dementia and aged 50+ at baseline (2008-09). Multinomial logistic regression models were fitted to estimate the relationship (relative risk ratios [RRR] and 95\% confidence intervals [CI]) between each factor, and the risk of CVD, dementia or both.

Results From the analytical sample, 13\% developed CVD, 4\% dementia and $1.5 \%$ both CVD and dementia during the eightyear follow-up period. After controlling for sociodemographic factors (age, sex, education and wealth), we found that hypertension $(\mathrm{RRR}=1.66,95 \% \mathrm{CI} 1.35-2.04)$, depressive symptoms $(\mathrm{RRR}=1.55$, 95\% CI 1.15-2.08), and obesity $(\mathrm{RRR}=1.37$, 95\% CI 1.03-1.83) were predictive of an increased risk of CVD; smoking $(\mathrm{RRR}=2.04$, 95\% CI 1.14-3.65) was associated with a higher risk of dementia; whilst smoking $(\mathrm{RRR}=2.34$, 95\% CI 1.03-5.27) and depressive symptoms $(\mathrm{RRR}=4.03$, 95\% CI 2.12-7.66) were predictive of developing both CVD and dementia. We found no associations between inflammatory markers, alcohol consumption or sedentary behaviour and these outcomes.

Conclusion Our findings indicate that smoking and depressive symptoms are associated with an increased risk of developing both CVD and dementia in later life, while obesity and hypertension are specific to CVD. There is relatively limited congruency across the predictive values of the 10 biopsychosocial risk factors investigated for these conditions. However, larger studies with longer periods of follow-up are necessary to extend these findings.

\section{OP18 WHAT EXPLAINS THE EFFECT OF EDUCATION ON CARDIOVASCULAR DISEASE? APPLYING MENDELIAN RANDOMISATION TO IDENTIFY THE CONSEQUENCES OF EDUCATION INEQUALITY} ${ }^{1} \mathrm{~L}$ Howe, ${ }^{2,6} \mathrm{~A}$ Dehghan. ${ }^{1} \mathrm{MRC}$ Integrative Epidemiology Unit, Population Health Sciences, University of Bristol, Bristol, UK; ${ }^{2}$ Department of Biostatistics and Epidemiology, Imperial College London, London, UK; ${ }^{3}$ NIHR Biomedical Research Centre, University Hospitals Bristol NHS Foundation Trust, Bristol, UK; ${ }^{4}$ Medical Research Council Population Health Research Unit, University of Oxford, Oxford, UK; ${ }^{5}$ Clinical Trial Service Unit and Epidemiological Studies Unit, University of Oxford, Oxford, UK; ${ }^{6}$ MRC-PHE Centre for Environment, School of Public Health, Imperial College London, London, UK; ${ }^{7}$ Department of Hygiene and Epidemiology, University of loannina, Ioannina, Greece

\subsection{6/jech-2019-SSMabstracts. 18}

Background Studies have demonstrated causal effects of educational attainment on cardiovascular disease (CVD). We aimed to investigate the role of body mass index (BMI), systolic blood pressure (SBP) and smoking in explaining the effect of education on risk of CVD outcomes triangulating across multivariable regression analysis of observational data and one- and 